\title{
DEFORMING CONVEX HYPERSURFACES BY THE $n$ TH ROOT OF THE GAUSSIAN CURVATURE
}

\author{
BENNETT CHOW
}

\section{Table of Contents}

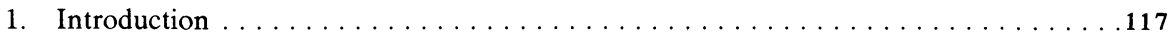

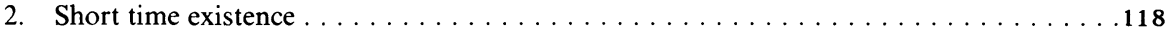

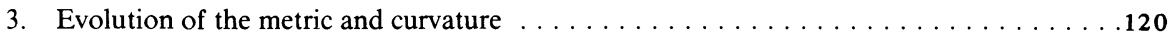

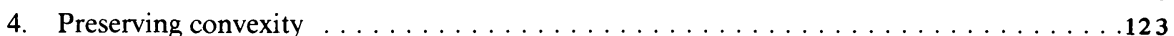

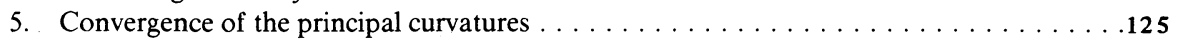

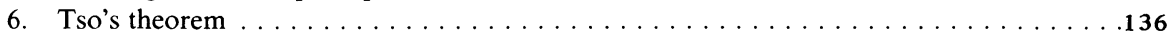

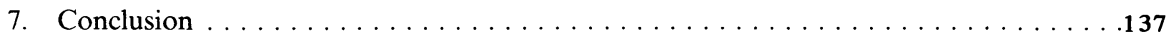

\section{Introduction}

Recently, G. Huisken [4] studied the deformation of convex hypersurfaces in $\mathbf{R}^{n+1}$ by their mean curvature vector. In particular, he proved the following:

1.1 Theorem. If the map $F_{0}: S^{n} \rightarrow M_{0} \subset \mathbf{R}^{n+1}$ represents a strictly convex smooth hypersurface in $\mathbf{R}^{n+1}, n \geqslant 2$, then the initial value problem

$$
\begin{gathered}
\frac{\partial F}{\partial t}(x, t)=-H(x, t) \cdot \nu(x, t), \\
F(x, 0)=F_{0}(x), \quad x \in S^{n},
\end{gathered}
$$

has a unique solution on a maximum finite time interval $[0, T)$ such that the $M_{t}$ 's converge to a point as $t \rightarrow T$. Here $H$ denotes the mean curvature and $\nu$ the outward normal of $M$. Moreover, if we let $\tilde{M}_{t}$ be $M_{t}$ rescaled by a homothetic expansion so that $\operatorname{Vol}\left(\tilde{M}_{t}\right)=\operatorname{Vol}\left(M_{0}\right)$, then as $t \rightarrow T$ the $\tilde{M}_{t}^{\prime}$ s converge to a smooth hypersurface $\tilde{M}_{T}$ in the $C^{\infty}$-topology. In fact $\tilde{M}_{T}$ is a round sphere.

The case $n=1$ is a theorem of M. Gage and R. Hamilton [2]. Thus Theorem 1.1 may be considered as a generalization of their theorem to dimensions $n \geqslant 2$. Another possible generalization of their theorem to higher dimensions is the deformation of a convex hypersurface by its Gaussian curvature $K$. 
Originally, Firey [1] considered this equation for surfaces in $\mathbf{R}^{3}$ as a model for the wearing of stones on a beach by water waves. Recently, Tso [9] studied the problem in arbitrary dimension. In particular, he proved

1.2 Theorem. If we replace (I) in Theorem 1.1 by

$$
\begin{gathered}
\frac{\partial F}{\partial t}(x, t)=-K(x, t) \cdot \nu(x, t), \\
F(x, 0)=F_{0}(x), \quad x \in S^{n},
\end{gathered}
$$

then the same conclusion as in Theorem 1.1 holds except that one does not know whether or not $\tilde{M}_{T}$ is a round sphere.

Inspired by these results, in this paper we prove

1.3 Theorem. If in Theorem 1.2 we replace (II) by

$$
\begin{gathered}
\frac{\partial F}{\partial t}(x, t)=-K(x, t)^{\beta} \cdot \nu(x, t), \\
F(x, 0)=F_{0}(x), \quad x \in S^{n},
\end{gathered}
$$

then for all $\beta>0$ the same conclusion as in Theorem 1.2 holds. Moreover, in the case $\beta=1 / n$ we can show that $\tilde{M}_{T}$ is a round sphere.

The first part of Theorem 1.3 is a straightforward generalization of Theorem 1.2. The bulk of this paper consists of showing the required estimates when $\beta=1 / n$. In the course of the proof of Theorem 1.3, it will become evident that the case $\beta=1 / n$ is borderline and the most natural from the point of view of the PDE's involved. Geometrically, $K^{1 / n}$ represents the geometric mean of the principal curvatures of $M$, whereas $H / n$ represents their arithmetic mean. Note that $K^{1 / n}$ is also scaled the same as $H$.

We also remark that in Theorem 1.3 , for $\beta \geqslant 1 / n$, there exist constants $0 \leqslant C(\beta) \leqslant 1 / n$ depending continuously on $\beta$ with $C(1 / n)=0$ and $\lim _{\beta \rightarrow \infty} C(\beta)=1 / n$ such that if the initial hypersurface $M_{0}$ satisfies $h_{i j} \geqslant$ $C(\beta) H g_{i j}$, then $\tilde{M}_{T}$ is a round sphere.

I would especially like to thank Professors G. Huisken, R. Hamilton and S.-T. Yau for many stimulating discussions and their encouragement. I would also like to thank Professors J. Jost and R. Schoen for their interest and encouragement. I am especially indebted to Professor Yau under whose advice this paper was written. This paper is dedicated to the memory of my sister Eleanor Chow.

\section{Short time existence}

If $M^{n} \subset \mathbf{R}^{n+1}$ is a hypersurface, the Gaussian curvature is given by $K=$ $\operatorname{det} h_{i j} / \operatorname{det} g_{i j}$, where $h_{i j}=-\left\langle\partial^{2} F / \partial x^{i} \partial x^{j}, \nu\right\rangle$ is the second fundamental form and $g_{i j}=\left\langle\partial F / \partial x^{i}, \partial F / \partial x^{j}\right\rangle$ is the induced metric on $M$ from $\mathbf{R}^{n+1}$ in the 
coordinate system $\left\{x^{i}\right\}$. We consider the evolution equation (III) for $\beta>0$. Note that even for strictly convex hypersurfaces, (III) is only weakly parabolic due to the fact that the diffeomorphism group of $S^{n}$ acts as a gauge group. However, short time existence follows as in [2] from an existence theorem of Hamilton [3] for evolution equations with an integrability condition.

2.1 Theorem. If $M_{0}$ is strictly convex, then (III) has a unique smooth solution $F(x, t)$ for short time.

Proof. We compute the symbol of the right-hand side (RHS) of (III). A variation $\tilde{F}$ of $F$ gives rise to variations $\tilde{g}_{i j}, \tilde{h}_{i j}$ and $\tilde{\nu}$ of $g_{i j}, h_{i j}$ and $\nu$. On the symbol level, we have $\tilde{h}_{i j}=-\xi_{i} \xi_{j}\langle\tilde{F}, \nu\rangle, \tilde{g}_{i j}=0$ and $\tilde{\nu}=0$. This is because $h_{i j}$ depends on the second derivatives of $F$ whereas $g_{i j}$ and $\nu$ depend only on the first derivatives. Therefore $\tilde{K}=K h_{i j}^{-1} \tilde{h}_{i j}=-K h_{i j}^{-1} \xi_{i} \xi_{j}\langle\tilde{F}, \nu\rangle$ and the symbol of the linearization of the RHS of (III) is given by

$$
\sigma D\left(-K^{\beta} \cdot \nu\right)(\xi) \tilde{F}=\beta K^{\beta}|\xi|_{h}^{2}\langle\tilde{F}, \nu\rangle \nu .
$$

Let $\pi_{M}$ and $\pi_{N}$ denote the projection of vectors in $\mathbf{R}^{n+1}$ onto the tangent and normal bundles of $M \subset \mathbf{R}^{n+1}$, respectively. Then $\sigma D\left(-K^{\beta} \nu\right)(\xi) \tilde{F}=$ $\beta K^{\beta}|\xi|_{h}^{2} \pi_{N}(\tilde{F})$ and $\pi_{M}\left(-K^{\beta} \nu\right)=0$ is our integrability condition. That is, for $\xi \neq 0$, the sequence

$$
\mathbf{R}^{n+1} \stackrel{\sigma D\left(-K^{\beta} \nu\right)(\xi)}{\longrightarrow} \mathbf{R}^{n+1} \stackrel{\pi_{M}}{\rightarrow} \mathbf{R}^{n+1}
$$

is exact. Therefore, as in [2], we may now apply Theorem 5.1 of [3] to conclude that there exists a unique solution of (III) for short time.

We remark that the second order partial differential operator $L=\beta K^{\beta} \square$, where $\square=h_{k l}^{-1} \nabla_{k} \nabla_{l}$, plays the same role for (III) as does the Laplace-Beltrami operator for (I). In particular, the second fundamental form and all curvatures formed from it satisfy equations of the form $\partial / \partial t=L+$ lower order. Thus, the fact that (III) may be written as $(\partial / \partial t) F(x, t)=(1 / n \beta) L F(x, t)$ explains the significance of the case $\beta=1 / n$; only in that case do the map $F$ and the second fundamental form satisfy equations which are equal in their highest order terms.

We now explain some notations which we will use for the rest of the paper. If $X$ and $Y$ are 1-forms and $s$ is a symmetric positive definite covariant 2-tensor on $M$, then $\langle X, Y\rangle_{s}$ denotes the inner product of $X$ and $Y$ with respect to the metric $s$. In local coordinates, $\langle X, Y\rangle_{s}=s_{i j}^{-1} X_{i} Y_{j}$. If $t$ is a contravariant 2-tensor, then we define $\langle X, Y\rangle_{t^{-1}}=t^{i j} X_{i} Y_{j}$. Thus $\langle X, Y\rangle_{t^{-1}}$ is well defined even for indefinite 2-tensors. If $A_{i k l}$ is a covariant 3-tensor, then

$$
\left|A_{i k l}\right|_{g, h}^{2}=g^{i j} h_{k m}^{-1} h_{l n}^{-1} A_{i k l} A_{j m n} .
$$


For future reference, we define $m=\left((H / n)^{n-1} g^{-1}-K h^{-1}\right)^{-1}$ and $e=$ $\left(h^{-1}-n g^{-1} / H\right)^{-1}$. By the preceding remarks, $\langle X, Y\rangle_{m}$ and $\langle X, Y\rangle_{e}$ are well defined.

\section{Evolution of the metric and curvature}

In this section we compute how the metric, second fundamental form, Gaussian and mean curvatures evolve. As in [4], we have

3.1 Lemma. The evolution equations for $g_{i j}, \nu$ and $h_{i j}$ are given by

$$
\begin{gathered}
\frac{\partial}{\partial t} g_{i j}=-2 K^{\beta} h_{i j}, \\
\frac{\partial}{\partial t} \nu=\nabla K^{\beta}, \\
\frac{\partial}{\partial t} h_{i j}=\nabla_{i} \nabla_{j} K^{\beta}-K^{\beta} h_{i k} h_{k j},
\end{gathered}
$$

where we suppress the raising of indices and the repetition of an index represents contraction with the metric.

Proof. This follows from straightforward computation as in $\S 3$ of [4]. (3) may be rewritten as

3.2 Lemma. We have

$$
\begin{aligned}
\frac{\partial}{\partial t} h_{i j}=\beta K^{\beta}\left(\square h_{i j}+\frac{\beta}{K^{2}} \nabla_{i}\right. & K \nabla_{j} K+\nabla_{i} h_{k l}^{-1} \nabla_{j} h_{k l} \\
& \left.+H h_{i j}-\left(n+\frac{1}{\beta}\right) h_{i k} h_{k j}\right)
\end{aligned}
$$

and equivalently

$$
\frac{\partial}{\partial t} h_{i j}=\beta K^{\beta}\left(\square h_{i j}+\frac{H^{2 n}}{n K^{2}} \nabla_{i}\left(\frac{K}{H^{n}}\right) \nabla_{j}\left(\frac{K}{H^{n}}\right)\right.
$$

$$
\begin{aligned}
& -\frac{1}{H^{2}} h_{k m}^{-1} h_{l n}^{-1}\left(H \nabla_{i} h_{m n}-\nabla_{i} H h_{m n}\right)\left(H \nabla_{j} h_{k l}-\nabla_{j} H h_{k l}\right) \\
& \left.+\left(\beta-\frac{1}{n}\right) \frac{1}{K^{2}} \nabla_{i} K \nabla_{j} K+H h_{i j}-\left(n+\frac{1}{\beta}\right) h_{i k} h_{k j}\right) .
\end{aligned}
$$

Proof. Recall that the Codazzi equations are

$$
\nabla_{i} h_{k l}=\nabla_{k} h_{i l}=\nabla_{l} h_{i k}
$$

and the rule for commuting two covariant derivatives acting on a 2-tensor $A_{k l}$ is given by

$$
\nabla_{i} \nabla_{j} A_{k l}-\nabla_{j} \nabla_{i} A_{k l}=R_{i j k m} A_{m l}+R_{i j l m} A_{m k}
$$


The relation between the Riemannian curvature tensor and the second fundamental form is given by Gauss' equation

$$
R_{i j k l}=h_{i k} h_{j l}-h_{i l} h_{j k} .
$$

We have $\nabla_{j} K=K h_{k l}^{-1} \nabla_{j} h_{k l}$ and by applying (6), (7) and (8) we obtain

$$
\begin{aligned}
\nabla_{i} \nabla_{j} K= & K h_{k l}^{-1} \nabla_{i} \nabla_{j} h_{k l}+K h_{m n}^{-1} \nabla_{i} h_{m n} h_{k l}^{-1} \nabla_{j} h_{k l}+K \nabla_{i} h_{k l}^{-1} \nabla_{j} h_{k l} \\
= & K h_{k l}^{-1} \nabla_{k} \nabla_{l} h_{i j}+\frac{1}{K} \nabla_{i} K \nabla_{j} K+K \nabla_{i} h_{k l}^{-1} \nabla_{j} h_{k l} \\
& +K h_{k l}^{-1}\left(\left(h_{i j} h_{k m}-h_{i m} h_{k j}\right) h_{m l}+\left(h_{i l} h_{k m}-h_{i m} h_{k l}\right) h_{m j}\right) \\
= & K\left(\square h_{i j}+\frac{1}{K^{2}} \nabla_{i} K \nabla_{j} K+\nabla_{i} h_{k l}^{-1} \nabla_{j} h_{k l}+H h_{i j}-n h_{i k} h_{k j}\right) .
\end{aligned}
$$

(3) now implies

(9) $\frac{\partial}{\partial t} h_{i j}=\beta K^{\beta-1} \nabla_{i} \nabla_{j} K+\beta(\beta-1) K^{\beta-2} \nabla_{i} K \nabla_{j} K-K^{\beta} h_{i k} h_{k j}$

and hence (4). We also have

$$
\begin{gathered}
\frac{1}{n K^{2}} \nabla_{i} K \nabla_{j} K+\nabla_{i} h_{k l}^{-1} \nabla_{j} h_{k l}=\frac{H^{2 n}}{n K^{2}} \nabla_{i}\left(\frac{K}{H^{n}}\right) \nabla_{j}\left(\frac{K}{H^{n}}\right)-\frac{n}{H^{2}} \nabla_{i} H \nabla_{j} H \\
+\frac{1}{H K}\left(\nabla_{i} H \nabla_{j} K+\nabla_{i} K \nabla_{j} H\right)-h_{k m}^{-1} h_{l n}^{-1} \nabla_{i} h_{m n} \nabla_{j} h_{k l}, \\
\frac{1}{H^{2}} h_{k m}^{-1} h_{l n}^{-1}\left(H \nabla_{i} h_{m n}-\nabla_{i} H h_{m n}\right)\left(H \nabla_{j} h_{k l}-\nabla_{j} H h_{k l}\right) \\
=h_{k m}^{-1} h_{l n}^{-1} \nabla_{i} h_{m n} \nabla_{j} h_{k l}-\frac{1}{H K}\left(\nabla_{i} H \nabla_{j} K+\nabla_{i} K \nabla_{j} H\right)+\frac{n}{H^{2}} \nabla_{i} H \nabla_{j} H
\end{gathered}
$$

so that

$$
\begin{aligned}
\frac{1}{n K^{2}} \nabla_{i} K & \nabla_{j} K+\nabla_{i} h_{k l}^{-1} \nabla_{j} h_{k l} \\
= & -\frac{1}{H^{2}} h_{k m}^{-1} h_{l n}^{-1}\left(H \nabla_{i} h_{m n}-\nabla_{i} H h_{m n}\right)\left(H \nabla_{j} h_{k l}-\nabla_{j} H h_{k l}\right) \\
& +\frac{H^{2 n}}{n K^{2}} \nabla_{i}\left(\frac{K}{H^{n}}\right) \nabla_{j}\left(\frac{K}{H^{n}}\right)
\end{aligned}
$$


Therefore

$$
\begin{aligned}
& \frac{\partial}{\partial t} h_{i j}=\beta K^{\beta}\left(\square h_{i j}\right.+\frac{H^{2 n}}{n K^{2}} \nabla_{i}\left(\frac{K}{H^{n}}\right) \nabla_{j}\left(\frac{K}{H^{n}}\right) \\
&-\frac{1}{H^{2}} h_{k m}^{-1} h_{l n}^{-1}\left(H \nabla_{i} h_{m n}-\nabla_{i} H h_{m n}\right)\left(H \nabla_{j} h_{k l}-\nabla_{j} H h_{k l}\right) \\
&\left.\quad+\left(\beta-\frac{1}{n}\right) \frac{1}{K^{2}} \nabla_{i} K \nabla_{j} K+H h_{i j}-\left(n+\frac{1}{\beta}\right) h_{i k} h_{k j}\right)
\end{aligned}
$$

which is equation (5).

When $\beta=1 / n,(4)$ may be rewritten as

$$
\begin{aligned}
\frac{\partial}{\partial t} h_{i j}= & \frac{1}{n} K^{1 / n}\left(\square h_{i j}+H h_{i j}-2 n h_{i k} h_{k j}\right) \\
& -\left(h_{k m}^{-1} h_{l n}^{-1} \nabla_{i} h_{m n} \nabla_{j} h_{k l}-\frac{1}{n} h_{m n}^{-1} \nabla_{i} h_{m n} h_{k l}^{-1} \nabla_{j} h_{k l}\right) .
\end{aligned}
$$

The gradient terms are negative, therefore we cannot show the inequality $h_{i j} \geqslant 0$ remains true for all time by applying the maximum principle for tensors of [3] directly to $h_{i j}$. In fact, the only thing we could prove is that the inequality $h_{i j} \leqslant 0$ is preserved under the evolution, which is the exact opposite of what we want to prove!

We now compute the evolution equations for $H$ and $K$.

3.3 Lemma. The mean and Gaussian curvatures satisfy the following evolution equations:

$$
\begin{gathered}
\frac{\partial}{\partial t} H=\beta K^{\beta}\left(\square H-\frac{1}{H^{2}}\left|H \nabla_{i} h_{k l}-\nabla_{i} H h_{k l}\right|_{g, h}^{2}+\frac{H^{2 n}}{n K^{2}}\left|\nabla\left(\frac{K}{H^{n}}\right)\right|^{2}\right. \\
\left.+\left(\beta-\frac{1}{n}\right) \frac{1}{K^{2}}|\nabla K|^{2}+H^{2}-\left(n-\frac{1}{\beta}\right)|A|^{2}\right), \\
\frac{\partial}{\partial t} K=\beta K^{\beta}\left(\square K+\frac{\beta-1}{K}|\nabla K|_{h}^{2}+\frac{1}{\beta} H K\right) .
\end{gathered}
$$

Proof. (10) follows directly from (1) and (5). By using (1) and (9), we have

$$
\begin{aligned}
\frac{\partial}{\partial t} K & =\frac{\partial}{\partial t}\left(\frac{\operatorname{det} h_{i j}}{\operatorname{det} g_{i j}}\right)=K\left(h_{i j}^{-1} \frac{\partial}{\partial t} h_{i j}-g^{i j} \frac{\partial}{\partial t} g_{i j}\right) \\
& =\beta K^{\beta}\left(\square K+\frac{\beta-1}{K}|\nabla K|_{h}^{2}+\frac{1}{\beta} H K\right)
\end{aligned}
$$

which is (11). 


\section{Preserving convexity}

Applying the maximum principle to (11) of Lemma 3.3, we have

4.1 Corollary. If $C>0$ is a constant and $K \geqslant C$ at $t=0$, then $K \geqslant C$ for all time. As a consequence, if $M_{t}$ is strictly convex at $t=0$, then it remains so.

In [4], Huisken, following an idea of Hamilton [3], looked at the function

$$
\frac{|A|^{2}-H^{2} / n}{H^{\delta}}=\frac{1}{H^{\delta}} \sum_{i \neq j}\left(\kappa_{i}-\kappa_{j}\right)^{2} \geqslant 0,
$$

where $\kappa_{1}, \cdots, \kappa_{n}$ are the principal curvatures of $M$. This quantity is scale invariant when $\delta=2$ and measures the difference of $M$ from being totally umbilic and hence a round sphere. We look at an analogous quantity which is more natural for our equation. By the arithmetic-geometric mean inequality, $(H / n)^{n}-K \geqslant 0$ on $M$ and equality holds at a point $p \in M$ if and only if $p$ is an umbilic point. The rest of this section consists of showing the inequality $K / H^{n} \geqslant C>0$ or, equivalently, $\left((H / n)^{n}-K\right) / H^{n} \leqslant C<(1 / n)^{n}$ is preserved under the evolution.

4.2 Lemma. We have the evolution equation

$$
\begin{aligned}
\frac{\partial}{\partial t}\left(\frac{K}{H^{n}}\right)=\beta K^{\beta}( & \square\left(\frac{K}{H^{n}}\right)+\frac{1}{K}\left(\frac{1}{n}-1\right)\left\langle\nabla K, \nabla\left(\frac{K}{H^{n}}\right)\right\rangle_{h} \\
& +\frac{n+1}{H}\left\langle\nabla H, \nabla\left(\frac{K}{H^{n}}\right)\right\rangle_{h}-\frac{H^{n-1}}{K}\left|\nabla\left(\frac{K}{H^{n}}\right)\right|^{2} \\
& +\frac{n K}{H^{n+3}}\left|H \nabla_{i} h_{k l}-\nabla_{i} H h_{k l}\right|_{g, h}^{2}+\frac{(\beta-1 / n)}{H^{n} K}|\nabla K|_{e}^{2} \\
& \left.+n\left(n-\frac{1}{\beta}\right) \frac{K}{H^{n+1}}\left(|A|^{2}-\frac{1}{n} H^{2}\right)\right),
\end{aligned}
$$

where $e=\left(h^{-1}-n / H \cdot g^{-1}\right)^{-1}$.

Proof. By (10) and (11) of Lemma 3.3

$$
\begin{aligned}
\frac{\partial}{\partial t}\left(\frac{K}{H^{n}}\right)=\beta K^{\beta}( & \square\left(\frac{K}{H^{n}}\right)+\frac{2 n}{H^{n+1}}\langle\nabla H, \nabla K\rangle_{h}-\frac{n(n+1) K}{H^{n+2}}|\nabla H|_{h}^{2} \\
& -\frac{n K}{H^{n+1}}\left(\frac{(\beta-1 / n)}{K^{2}}|\nabla K|^{2}+\frac{H^{2 n}}{n K^{2}}\left|\nabla\left(\frac{K}{H^{n}}\right)\right|^{2}\right. \\
& \left.-\frac{1}{H^{2}}\left|H \nabla_{i} h_{k l}-\nabla_{i} H \cdot h_{k l}\right|_{g, h}^{2}+H^{2}-\left(n-\frac{1}{\beta}\right)|A|^{2}\right) \\
& \left.+\frac{1}{H^{n}}\left(\frac{(\beta-1)}{K}|\nabla K|_{h}^{2}+\frac{1}{\beta} H K\right)\right) .
\end{aligned}
$$


Then the equality

$$
\begin{aligned}
\frac{2 n}{H^{n+1}}\langle\nabla H, \nabla K\rangle_{h}-\frac{n(n+1) K}{H^{n+2}}|\nabla H|_{h}^{2}+\frac{(1 / n-1)}{H^{n} K}|\nabla K|_{h}^{2} \\
=\frac{(1 / n-1)}{K}\left\langle\nabla K, \nabla\left(\frac{K}{H^{n}}\right)\right\rangle_{h}+\frac{n+1}{H}\left\langle\nabla H, \nabla\left(\frac{K}{H^{n}}\right)\right\rangle_{h}
\end{aligned}
$$

implies

$$
\begin{aligned}
\frac{\partial}{\partial t}\left(\frac{K}{H^{n}}\right)=\beta K^{\beta}( & \square\left(\frac{K}{H^{n}}\right)+\frac{(1 / n-1)}{K}\left\langle\nabla K, \nabla\left(\frac{K}{H^{n}}\right)\right\rangle_{h} \\
& +\frac{n+1}{H}\left\langle\nabla H, \nabla\left(\frac{K}{H^{n}}\right)\right\rangle_{h}-\frac{H^{n-1}}{K}\left|\nabla\left(\frac{K}{H^{n}}\right)\right|^{2} \\
& +\frac{n K}{H^{n+3}}\left|H \nabla_{i} h_{k l}-\nabla_{i} H h_{k l}\right|_{g, h}^{2}+\frac{(\beta-1 / n)}{H^{n} K}|\nabla K|_{e}^{2} \\
& \left.+n\left(n-\frac{1}{\beta}\right) \frac{K}{H^{n+1}}\left(|A|^{2}-\frac{1}{n} H^{2}\right)\right),
\end{aligned}
$$

which is (12).

In the case $\beta=1 / n$, the last two terms of (12) drop out, and by applying the maximum principle we have

4.3 Corollary. In the case $\beta=1 / n$, if $K / H^{n} \geqslant C>0$ at $t=0$, then it remains so.

As remarked earlier, $|A|^{2}-H^{2} / n \geqslant 0$, so that when $\beta>1 / n$ the zeroth order term on the RHS of (12) is nonnegative and hence a good term. Now, by Lemma 2.3(ii) of [4] we have the estimate

$$
\left|H \nabla_{i} h_{k l}-\nabla_{i} H h_{k l}\right|^{2} \geqslant \frac{1}{2} \varepsilon^{2} H^{2}|\nabla H|^{2}
$$

provided $h_{i j} \geqslant \varepsilon H g_{i j}$. Since $h_{i j} \leqslant H g_{i j}$, we have

$$
\begin{aligned}
\frac{n K}{H^{n+3}}\left|H \nabla_{i} h_{k l}-\nabla_{i} H \cdot h_{k l}\right|_{g, h}^{2} & \geqslant \frac{n K}{H^{n+1}}\left|H \nabla_{i} h_{k l}-\nabla_{i} H \cdot h_{k l}\right|^{2} \\
& \geqslant \frac{n \varepsilon^{2}}{2} \frac{K}{H^{n+3}}|\nabla H|^{2} .
\end{aligned}
$$

Choosing $C(\beta)$ close enough to $1 / n$, we have

$$
\left|h^{-1}-\frac{n}{H} g^{-1}\right| \leqslant \frac{\varepsilon^{2}}{2 n \beta} \frac{1}{H},
$$


provided $h_{i j} \geqslant C(\beta) H g_{i j}$. Therefore

$$
\begin{aligned}
\left.\left|\frac{(\beta-1 / n)}{H^{n} K}\right| \nabla K\right|_{e} ^{2} \mid & \leqslant \frac{(\beta-1 / n)}{H^{n} K}|\nabla K|^{2}\left|h^{-1}-\frac{n}{H} g^{-1}\right| \\
& \leqslant \frac{n \varepsilon^{2}}{2} \frac{K}{H^{n+3}}|\nabla H|^{2}+\text { terms linear in } \nabla\left(\frac{K}{H^{n}}\right),
\end{aligned}
$$

and hence

$$
\begin{array}{r}
\frac{n K}{H H^{n+3}}\left|H \cdot \nabla_{i} h_{k l}-\nabla_{i} H \cdot h_{k l}\right|_{g, h}^{2}+\frac{(\beta-1 / n)}{H^{n} K}|\nabla K|_{e}^{2} \geqslant 0 \\
\text { modulo } \nabla\left(\frac{K}{H^{n}}\right) \text { terms. }
\end{array}
$$

Therefore, applying the maximum principle and $\left(13^{\prime}\right)$ to $(12)$, we have

4.4 Corollary. For $\beta \geqslant 1 / n$, there exist constants $C(\beta)<1 / n$ depending only on $n$ and $\beta$ with $C(1 / n)=0$ and $\lim _{\beta \rightarrow \infty} C(\beta)=1 / n$ such that if $h_{i j} \geqslant C(\beta) H g_{i j}$ at $t=0$, then the inquality $K / H^{n} \geqslant C>0$ is preserved under the evolution.

\section{Convergence of the principal curvatures}

In this section we will prove the main estimate which will show that the shape of $M_{t}$ approaches that of a round sphere as $t \rightarrow T$. Let $f=1 / n^{n}-K / H^{n}$ and $f_{\sigma}=H^{\sigma} f$. Then as remarked in $\S 4, f \geqslant 0$ with equality holding only at umbilic points.

5.1 Theorem. If $\beta=1 / n$, then there exist constants $\sigma_{0}>0$ and $C_{0}<\infty$ depedning only on $M_{0}$ such that

$$
\frac{1}{n^{n}}-\frac{K}{H^{n}} \leqslant C_{0} H^{-\sigma_{0}}
$$

for all time. If $\beta>1 / n$, then inequality (14) is actually preserved under the evolution provided $h_{i j} \geqslant C(\beta) H g_{i j}$ at $t=0$ where $C(\beta)<1 / n$ is sufficiently close to $1 / n$ and where $\sigma_{0}$ now depends also on $\beta$.

The rest of this section will consist of proving Theorem 5.1.

5.2 Lemma. If $\beta=1 / n$, then there exist constants $\sigma_{1}>0, C_{1}>0$ and $C_{2}<\infty$ depending only on $M_{0}$ such that for $\sigma<\sigma_{1}$,

$$
\frac{\partial f_{\sigma}}{\partial t} \leqslant \frac{1}{n} K^{1 / n}\left(\square f_{\sigma}-C_{1} H^{\sigma-3}|\nabla H|^{2}+C_{2} H^{-1-\sigma}\left|\nabla f_{\sigma}\right|^{2}+\sigma H f_{\sigma}\right) .
$$


If $\beta>1 / n$, then there exist constants $\sigma_{1}(\beta)>0, C(\beta)<1 / n, C_{1}>0$ and $C_{2}(\beta)<\infty$ depending only on $\beta$ and $M_{0}$ such that for $\sigma<\sigma_{1}(\beta)$

$$
\frac{\partial f_{\sigma}}{\partial t} \leqslant \beta K^{\beta}\left(\square f_{\sigma}-C_{1} H^{\sigma-3}|\nabla H|^{2}+C_{2}(\beta) H^{-1-\sigma}\left|\nabla f_{\sigma}\right|^{2}\right)
$$

provided $h_{i j} \geqslant C(\beta) H g_{i j}$ at $t=0$.

Proof. By (10) and (12),

$$
\begin{aligned}
\frac{\partial f}{\partial t}=\beta K^{\beta}(\square f & +\frac{(1 / n-1)}{K}\langle\nabla K, \nabla f\rangle+\frac{n+1}{H}\langle\nabla H, \nabla f\rangle+\frac{H^{n-1}}{K}|\nabla f|^{2} \\
& -\frac{n K}{H^{n+3}}\left|H \nabla_{i} h_{k l}-\nabla_{i} H h_{k l}\right|_{g, h}^{2}-\frac{(\beta-1 / n)}{H^{n} K}|\nabla K|_{e}^{2} \\
& \left.-n\left(n-\frac{1}{\beta}\right) \frac{K}{H^{n+1}}\left(|A|^{2}-\frac{1}{n} H^{2}\right)\right), \\
\frac{\partial H}{\partial t} \leqslant & \beta K^{\beta}\left(\square H+\frac{(\beta-1 / n)}{K^{2}}|\nabla K|^{2}+H^{2}-\left(n-\frac{1}{\beta}\right)|A|^{2}\right) .
\end{aligned}
$$

Therefore

$$
\begin{aligned}
& \frac{\partial f_{\sigma}}{\partial t}=\frac{\partial}{\partial t}\left(H^{\sigma} f\right)=H^{\sigma} \frac{\partial f}{\partial t}+\sigma H^{\sigma-1} f \frac{\partial H}{\partial t} \\
& \leqslant \beta K^{\beta}\left[\square f_{\sigma}-2 \sigma H^{\sigma-1}\langle\nabla H, \nabla f\rangle_{h}-\sigma(\sigma-1) H^{\sigma-2} f|\nabla H|_{h}^{2}\right. \\
& +H^{\sigma}\left(-\frac{(1 / n-1) H^{n}}{K}|\nabla f|_{h}^{2}+\frac{2}{H}\langle\nabla H, \nabla f\rangle_{h}+\frac{H^{n-1}}{K}|\nabla f|^{2}\right. \\
& -\frac{3 C_{1}}{H^{3}}|\nabla H|^{2}+\frac{C_{3}(\beta-1 / n)}{H^{n+2} K}\left(|A|^{2}-\frac{1}{n} H^{2}\right)^{1 / 2}|\nabla K|^{2} \\
& \left.\left.-n\left(n-\frac{1}{\beta}\right) \frac{K}{H^{n+1}}\left(|A|^{2}-\frac{1}{n} H^{2}\right)\right)+\sigma H f_{\sigma}-\sigma\left(n-\frac{1}{\beta}\right) \frac{|A|^{2}}{H} f_{\sigma}\right],
\end{aligned}
$$

where we used (13) and $C_{1}>0$ and $C_{3}<\infty$ are constants depending only on $M_{0}$.

We first consider the case $\beta=1 / n$. Then (17) gives

$$
\begin{aligned}
\frac{\partial f_{\sigma}}{\partial t} \leqslant \frac{1}{n} K^{1 / n}\left[\square f_{\sigma}\right. & -2 \sigma H^{\sigma-1}\langle\nabla H, \nabla f\rangle_{h}-\sigma(\sigma-1) H^{\sigma-2} f|\nabla H|_{h}^{2} \\
& +H^{\sigma}\left(-\frac{(1 / n-1) H^{n}}{K}|\nabla f|_{h}^{2}+\frac{2}{H}\langle\nabla H, \nabla f\rangle_{h}\right. \\
& \left.\left.+\frac{H^{n-1}}{K}|\nabla f|^{2}-\frac{3 C_{1}}{H}|\nabla H|^{2}\right)+\sigma H f_{\sigma}\right] .
\end{aligned}
$$


We have the equalities

$$
\begin{gathered}
|\nabla f|^{2}=H^{-2 \sigma}\left|\nabla f_{\sigma}\right|^{2}-2 \sigma H^{-1-2 \sigma} f_{\sigma}\left\langle\nabla H, \nabla f_{\sigma}\right\rangle+\sigma^{2} H^{-2-2 \sigma} f_{\sigma}^{2}|\nabla H|^{2}, \\
\langle\nabla H, \nabla f\rangle=H^{-\sigma}\left\langle\nabla H, \nabla f_{\sigma}\right\rangle-\frac{\sigma f}{H}|\nabla H|^{2}
\end{gathered}
$$

and the inequality

$$
\langle a, b\rangle \leqslant \frac{\eta}{2}|a|^{2}+\frac{1}{2 \eta}|b|^{2}, \quad \eta>0 .
$$

Applying (19) and (20) with $a=\nabla H, b=\nabla f_{\sigma}$ to (18) implies (15) for sufficiently small $\sigma$.

When $\beta>1 / n$, the zeroth order term on the RHS of (17) is nonpositive for $\sigma$ sufficiently small depending on $\beta$. Also, if $h_{i j} \geqslant C(\beta) H g_{i j}$ for $C(\beta)<1 / n$ sufficiently close to $1 / n$, then

$$
C_{3} \frac{(\beta-1 / n)}{H^{n+2} K}\left(|A|^{2}-\frac{1}{n} H^{2}\right)^{1 / 2}|\nabla K|^{2} \leqslant \frac{C_{1}}{H^{3}}|\nabla H|^{2}
$$

modulo controllable $|\nabla f|^{2}$ and $\langle\nabla H, \nabla f\rangle$ terms. Therefore, by applying (19) and (20) to (17) we have (16) provided $h_{i j} \geqslant C(\beta) H g_{i j}$ at $t=0$ and where the constants now depend also on $\beta$. This completes the proof of Lemma 5.2.

When $\beta>1 / n$, Theorem 5.1 is a direct consequence of (16) of Lemma 5.2 and the maximum principle. Therefore, in proving Theorem 5.1, we will restrict ourselves to the case $\beta=1 / n$. Then, (15) has bad (positive) zeroth order terms and we may not apply the maximum principle directly. As in [4], we prove a lemma which will allow us to bound the $L^{p}$-norms of $f_{\sigma}$. The supremum norm bound of $f_{\sigma}$ will then follow from the Sobolev inequality for submanifolds of $\mathbf{R}^{n}$ and an iteration type argument.

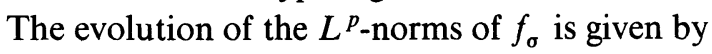

5.3 Lemma. There exist constants $0<C_{5}, C_{6}, C_{7}, C_{7^{\prime}}<\infty$ depending only on $M_{0}$ such that for $p \geqslant C_{5}$,

$$
\frac{\partial}{\partial t} \int f_{\sigma}^{p} \leqslant \int-C_{6} p^{2} f_{\sigma}^{p-2}\left|\nabla f_{\sigma}\right|^{2}-C_{7} p H^{\sigma-2} f_{\sigma}^{p-1}|\nabla H|^{2}+C_{7^{\prime}} \sigma p H^{2} f_{\sigma}^{p} .
$$

\section{Proof. By (15),}

$$
\begin{aligned}
\frac{\partial}{\partial t} f_{\sigma}^{p} & =p f_{\sigma}^{p-1} \frac{\partial f_{\sigma}}{\partial t} \\
& \leqslant \frac{p}{n} f_{\sigma}^{p-1} K^{1 / n}\left(\square f_{\sigma}-C_{1} H^{\sigma-3}|\nabla H|^{2}+C_{2} H^{-1-\sigma}\left|\nabla f_{\sigma}\right|^{2}+\sigma H f_{\sigma}\right) .
\end{aligned}
$$

Then

$$
\square f_{\sigma}^{p}=p f_{\sigma}^{p-1} \square f_{\sigma}+p(p-1) f_{\sigma}^{p-2}\left|\nabla f_{\sigma}\right|_{h}^{2}
$$


implies

$$
\begin{array}{r}
\frac{\partial}{\partial t} f_{\sigma}^{p} \leqslant \frac{1}{n} K^{1 / n}\left(\square f_{\sigma}^{p}-p(p-1) f_{\sigma}^{p-2}\left|\nabla f_{\sigma}\right|_{h}^{2}-C_{1} p f_{\sigma}^{p-1} H^{\sigma-3}|\nabla H|^{2}\right. \\
\left.+C_{2} p f_{\sigma}^{p-1} H^{-1-\sigma}\left|\nabla f_{\sigma}\right|^{2}+\sigma p H f_{\sigma}^{p}\right)
\end{array}
$$

and hence, by integrating,

$$
\begin{aligned}
\frac{\partial}{\partial t} \int f_{\sigma}^{p} \leqslant \frac{1}{n} \int K^{1 / n}\left(\square f_{\sigma}^{p}-\right. & \frac{1}{2} p(p-1) f_{\sigma}^{p-2}\left|\nabla f_{\sigma}\right|_{h}^{2} \\
& \left.-C_{1} p f_{\sigma}^{p-1} H^{\sigma-3}|\nabla H|^{2}+\sigma p H f_{\sigma}^{p}\right)
\end{aligned}
$$

provided $p \geqslant C_{4}$, where $C_{4}$ is a constant depending only on $M_{0}$, and we ignored the negative term coming from differentiation of the volume form. Now

$$
\begin{aligned}
\int K^{1 / n} \square f_{\sigma}^{p} & =\int K^{1 / n} h_{i j}^{-1} \nabla_{i} \nabla_{j} f_{\sigma}^{p} \\
& =-\frac{1}{n} \int K^{1 / n-1} h_{i j}^{-1} \nabla_{i} K \nabla_{j} f_{\sigma}^{p}-\int K^{1 / n} \nabla_{i} h_{i j}^{-1} \nabla_{j} f_{\sigma}^{p}
\end{aligned}
$$

and by the Codazzi equations,

$$
\nabla_{i} h_{i j}^{-1}=-h_{i k}^{-1} h_{j l}^{-1} \nabla_{i} h_{k l}=-h_{j l}^{-1} h_{i k}^{-1} \nabla_{l} h_{i k}=-h_{j l}^{-1} \frac{1}{K} \nabla_{l} K .
$$

Therefore

$$
\int K^{1 / n} \square f_{\sigma}^{p}=p\left(1-\frac{1}{n}\right) \int K^{1 / n-1} f_{\sigma}^{p-1}\left\langle\nabla K, \nabla f_{\sigma}\right\rangle_{h} .
$$

The equality

$$
\begin{aligned}
\left\langle\nabla K, \nabla f_{\sigma}\right\rangle_{h} & =-H^{n}\left\langle\nabla f, \nabla f_{\sigma}\right\rangle_{h}+\frac{n K}{H}\left\langle\nabla H, \nabla f_{\sigma}\right\rangle_{h} \\
& =-H^{n-\sigma}\left|\nabla f_{\sigma}\right|_{h}^{2}+\sigma H^{n-1-\sigma} f_{\sigma}\left\langle\nabla H, \nabla f_{\sigma}\right\rangle_{h}+\frac{n K}{H}\left\langle\nabla H, \nabla f_{\sigma}\right\rangle_{h}
\end{aligned}
$$

then implies

$$
\int K^{1 / n} \square f_{\sigma}^{p} \leqslant C_{8} p \int \frac{1}{H} f_{\sigma}^{p-1}|\nabla H|\left|\nabla f_{\sigma}\right|,
$$

where $C_{8}<\infty$ is a constant depending only on $M_{0}$. By applying (20) to (21) and (23), we have

$$
\frac{\partial}{\partial t} \int f_{\sigma}^{p} \leqslant \int-C_{6} p^{2} f_{\sigma}^{p-2}\left|\nabla f_{\sigma}\right|^{2}-C_{7} p H^{\sigma-2} f_{\sigma}^{p-1}|\nabla H|^{2}+C_{7^{\prime}} \sigma p H^{2} f_{\sigma}^{p},
$$

where $0<C_{5}, C_{6}, C_{7}, C_{7^{\prime}}<\infty$ depend only on $M_{0}$ provided $p \geqslant C_{5}$. This proves Lemma 5.3.

We now prove the lemma which will enable us to bound the $L^{p}$-norms of $f_{\sigma}$ for $p$ sufficiently large and $\sigma$ sufficiently small. 
5.4 Lemma. There exist positive constants $C_{18}, C_{19}, C_{20}$ and $C_{21}$ depending only on $M_{0}$ such that for any $0 \leqslant \sigma \leqslant 1, \eta>0$ and $p \geqslant C_{18}$,

$$
\int H^{2} f_{\sigma}^{p} \leqslant\left(C_{19} p \eta+C_{20}\right) \int H^{\sigma-2} f_{\sigma}^{p-1}|\nabla H|^{2}+C_{21} p \eta^{-1} \int f_{\sigma}^{p-2}\left|\nabla f_{\sigma}\right|^{2} .
$$

Proof. Recall $f_{\sigma}=\left(\left(1 / n^{n}\right) H^{n}-K\right) / H^{n-\sigma}$ so that

$$
\begin{aligned}
& \square f_{\sigma}=\frac{\square\left(\left(1 / n^{n}\right) H^{n}-K\right)}{H^{n-\sigma}}-\frac{n-\sigma}{H} f_{\sigma} \square H+\frac{(n-\sigma)(n+1-\sigma)}{H^{2}} f_{\sigma}|\nabla H|_{h}^{2} \\
& \quad-\frac{2(n-\sigma)}{H^{n+1-\sigma}}\left\langle\nabla H, \nabla\left(\frac{1}{n^{n}} H^{n}-K\right)\right\rangle_{h} .
\end{aligned}
$$

We have

$$
\begin{aligned}
& \begin{aligned}
& \square\left(\frac{1}{n^{n}} H^{n}-K\right)=\frac{1}{n^{n}}\left(n H^{n-1} h_{i j}^{-1} \nabla_{i} \nabla_{j} H+n(n-1) H^{n-2}|\nabla H|_{h}^{2}\right) \\
& \quad-K\left(h_{k l}^{-1} h_{i j}^{-1} \nabla_{i} \nabla_{j} h_{k l}+\frac{1}{K^{2}}|\nabla K|_{h}^{2}+h_{i j}^{-1} \nabla_{i} h_{k l}^{-1} \nabla_{j} h_{k l}\right) \\
&(25) \quad h_{i j}^{-1}\left(\left(\frac{H}{n}\right)^{n-1} g^{k l}-K h_{k l}^{-1}\right) \nabla_{i} \nabla_{j} h_{k l}+\frac{n-1}{n^{n-1}} H^{n-2}|\nabla H|_{h}^{2} \\
& \quad-\frac{1}{K}|\nabla K|_{h}^{2}-K h_{i j}^{-1} \nabla_{i} h_{k l}^{-1} \nabla_{j} h_{k l}
\end{aligned}
\end{aligned}
$$

and by applying the Codazzi equations,

$$
\nabla_{i} \nabla_{j} h_{k l}=\nabla_{k} \nabla_{l} h_{i j}+\left(h_{i j} h_{k m}-h_{i m} h_{k j}\right) h_{m l}+\left(h_{i l} h_{k m}-h_{i m} h_{k l}\right) h_{m j} .
$$

Since

$$
\begin{aligned}
& h_{i j}^{-1}\left(\left(\frac{H}{n}\right)^{n-1} g^{k l}-K h_{k l}^{-1}\right)\left(\left(h_{i j} h_{k m}-h_{i m} h_{k j}\right) h_{m l}+\left(h_{i l} h_{k m}-h_{i m} h_{k l}\right) h_{m j}\right) \\
& =\left(\left(\frac{H}{n}\right)^{n-1} g^{k l}-K h_{k l}^{-1}\right)\left(n h_{k m} h_{m l}-H h_{k l}\right)=n\left(\frac{H}{n}\right)^{n-1}\left(|A|^{2}-\frac{1}{n} H^{2}\right),
\end{aligned}
$$

(25) may be rewritten as

$$
\begin{aligned}
\square\left(\frac{1}{n^{n}} H^{n}-K\right)= & h_{i j}^{-1}\left(\left(\frac{H}{n}\right)^{n-1} g^{k l}-K h_{k l}^{-1}\right) \nabla_{k} \nabla_{l} h_{i j} \\
& +n\left(\frac{H}{n}\right)^{n-1}\left(|A|^{2}-\frac{1}{n} H^{2}\right)+\frac{n-1}{n^{n-1}} H^{n-2}|\nabla H|_{h}^{2} \\
& -\frac{1}{K}|\nabla K|_{h}^{2}-K h_{i j}^{-1} \nabla_{i} h_{k l}^{-1} \nabla_{j} h_{k l} .
\end{aligned}
$$


(24) and (26) imply

$$
\begin{gathered}
\square f_{\sigma}=\frac{1}{H^{n-\sigma}}\left[h_{i j}^{-1}\left(\left(\frac{H}{n}\right)^{n-1} g^{k l}-K h_{k l}^{-1}\right), \nabla_{k} \nabla_{l} h_{i j}+n\left(\frac{H}{n}\right)^{n-1}\left(|A|^{2}-\frac{1}{n} H^{2}\right)\right. \\
\left.\quad+\frac{n-1}{n^{n-1}} H^{n-2}|\nabla H|_{h}^{2}-\frac{1}{K}|\nabla K|_{h}^{2}-K h_{i j}^{-1} \nabla_{i} h_{k l}^{-1} \nabla_{j} h_{k l}\right] \\
\text { (27) } \quad-\frac{n-\sigma}{H} f_{\sigma} \square H+\frac{(n-\sigma)(n-1-\sigma)}{H^{2}} f_{\sigma}|\nabla H|_{h}^{2} \\
-\frac{2(n-\sigma)}{H^{n+1-\sigma}}\left\langle\nabla H, \nabla\left(\frac{1}{n^{n}} H^{n}-K\right)\right\rangle_{h} .
\end{gathered}
$$

We have

$$
\int K^{1 / n} \square f_{\sigma}^{p}=p \int K^{1 / n} f_{\sigma}^{p-1} \square f_{\sigma}+p(p-1) \int K^{1 / n} f_{\sigma}^{p-2}\left|\nabla f_{\sigma}\right|_{h}^{2}
$$

which, together with (22), implies

$$
\begin{aligned}
0=(p-1) & \int K^{1 / n} f_{\sigma}^{p-2}\left|\nabla f_{\sigma}\right|_{h}^{2} \\
& \quad-\left(1-\frac{1}{n}\right) \int K^{1 / n-1} f_{\sigma}^{p-1}\left\langle\nabla K, \nabla f_{\sigma}\right\rangle_{h}+\int K^{1 / n} f_{\sigma}^{p-1} \square f_{\sigma} .
\end{aligned}
$$

Multiplying (27) by $K^{1 / n} f_{\sigma}^{p-1}$ and integrating, we have

$$
\begin{aligned}
\int K^{1 / n} f_{\sigma}^{p-1} \square f_{\sigma} & =-\int\left(\left(\frac{H}{n}\right)^{n-1} g^{k l}-K h_{k l}^{-1}\right) \nabla_{k}\left(\frac{K^{1 / n} f_{\sigma}^{p-1}}{H^{n-\sigma}}\right) h_{i j}^{-1} \nabla_{l} h_{i j} \\
& -\int \frac{K^{1 / n} f_{\sigma}^{p-1}}{H^{n-\sigma}}\left(\left(\frac{H}{n}\right)^{n-1} g^{k l}-K h_{k l}^{-1}\right) \nabla_{k} h_{i j}^{-1} \nabla_{l} h_{i j} \\
& -\frac{n-1}{n^{n-1}} \int \frac{K^{1 / n} f_{\sigma}^{p-1}}{H^{2-\sigma}} g^{k l} \nabla_{k} H \cdot h_{i j}^{-1} \nabla_{l} h_{i j} \\
& +\int \frac{K^{1 / n} f_{\sigma}^{p-1}}{H^{n-\sigma}}\left(n\left(\frac{H}{n}\right)^{n-1}\left(|A|^{2}-\frac{1}{n} H^{2}\right)\right. \\
& \left.+\frac{n-1}{n^{n-1}} H^{n-2}|\nabla H|_{h}^{2}-\frac{1}{K}|\nabla K|_{h}^{2}-K h_{i j}^{-1} \nabla_{i} h_{k l}^{-1} \nabla_{j} h_{k l}\right) \\
& +\int K^{1 / n} f_{\sigma}^{p-1}\left(-\frac{n-\sigma}{H} f_{\sigma} \square H+\frac{(n-\sigma)(n+1-\sigma)}{H^{2}} f_{\sigma}|\nabla H|_{h}^{s}\right. \\
& \left.-\frac{2(n-\sigma)}{H^{n+1-\sigma}}\left\langle\nabla H, \nabla\left(\frac{1}{n^{n}} H^{n}-K\right)\right\rangle_{h}\right),
\end{aligned}
$$


where we integrated by parts and used $\nabla_{k}\left(K h_{k l}^{-1}\right)=0$. By applying the Codazzi equations we have

$$
\int a \square b=-\int\langle\nabla a, \nabla b\rangle_{h}+\int \frac{a}{K}\langle\dot{\nabla} K, \nabla b\rangle_{h}
$$

for any smooth functions $a$ and $b$ on $M$. This and the equality

$$
|\nabla K|_{h}^{2}=H^{2 n}|\nabla f|_{h}^{2}-2 n H^{n-1} K\langle\nabla H, \nabla f\rangle_{h}+\frac{n^{2} K^{2}}{H^{2}}|\nabla H|_{h}^{2}
$$

applied to (29) imply

$$
\begin{aligned}
& \int K^{1 / n} f_{\sigma}^{p-1} \square f_{\sigma}=-\int\left(\left(\frac{H}{n}\right)^{n-1} g^{k l}-K h_{k l}^{-1}\right) \nabla_{k}\left(\frac{K^{1 / n} f_{\sigma}^{p-1}}{H^{n-\sigma}}\right) \frac{\nabla_{l} K}{K} \\
& -\frac{1}{n^{n-1}} \int \frac{K^{1 / n} f_{\sigma}^{p-1}}{H^{1-\sigma}}\left\langle\nabla_{k} h_{i j}^{-1}, \nabla_{k} h_{i j}\right\rangle \\
& +\frac{n-1}{n^{n-1}} \int H^{n-2+\sigma} K^{1 / n-1} f_{\sigma}^{p-1}\langle\nabla H, \nabla f\rangle \\
& \text { (30) } \quad+\frac{n-1}{n^{n-1}} \int \frac{K^{1 / n} f_{\sigma}^{p-1}}{H^{2-\sigma}}|\nabla H|_{e}^{2}+\frac{1}{n^{n-2}} \int \frac{K^{1 / n} f_{\sigma}^{p-1}}{H^{1-\sigma}}\left(|A|^{2}-\frac{1}{n} H^{2}\right) \\
& -\int \frac{K^{1 / n-1} f_{\sigma}^{p-1}}{H^{n-\sigma}}\left(H^{2 n}|\nabla f|_{h}^{2}-2 n H^{n-1} K\langle\nabla H, \nabla f\rangle_{h}+\frac{n^{2} K^{2}}{H^{2}}|\nabla H|_{h}^{2}\right) \\
& +(n-\sigma) \int \frac{1}{H}\left\langle\nabla H, \nabla\left(K^{1 / n} f_{\sigma}^{p}\right)\right\rangle_{h}-(n-\sigma) \int \frac{K^{1 / n} f_{\sigma}^{p}}{H^{2}}|\nabla H|_{h}^{2} \\
& -(n-\sigma) \int \frac{K^{1 / n} \cdot f_{\sigma}^{p}}{H K}\langle\nabla K, \nabla H\rangle_{h} \\
& +\int K^{1 / n} f_{\sigma}^{p-1} \frac{(n-\sigma)}{H}\left(\frac{1-n+\sigma}{H} f_{\sigma}|\nabla H|_{h}^{2}-2\left\langle\nabla H, \nabla f_{\sigma}\right\rangle_{h}\right) .
\end{aligned}
$$

We have

$$
\frac{1}{H^{2}}\left|H \nabla_{i} h_{k l}-\nabla_{i} H \cdot h_{k l}\right|_{g, h}^{2}
$$

$$
=-\left\langle\nabla_{i} h_{k l}^{-1}, \nabla_{i} h_{k l}\right\rangle \frac{2 H^{n-1}}{K}\langle\nabla H, \nabla f\rangle-\frac{n}{H^{2}}|\nabla H|^{2} .
$$


Applying $\left(29^{\prime}\right)$ and $\left(30^{\prime}\right)$ to (30) gives

$$
\begin{aligned}
& \int K^{1 / n} f_{\sigma}^{p-1} \square f_{\sigma}=(p-1) \int H^{\sigma} K^{1 / n-1} f_{\sigma}^{p-2}\left\langle\nabla f, \nabla f_{\sigma}\right\rangle_{m} \\
& -n(p-1) \int \frac{K^{1 / n} f_{\sigma}^{p-2}}{H^{n+1-\sigma}}\left\langle\nabla H, \nabla f_{\sigma}\right\rangle_{m} \\
& -\frac{1}{n} \int \frac{K^{1 / n} f_{\sigma}^{p-1}}{H^{n-\sigma}}\left(H^{2 n}|\nabla f|_{m}^{2}-2 n H^{n-1} K\langle\nabla H, \nabla f\rangle_{m}+\frac{n^{2} K^{2}}{H^{2}}|\nabla H|_{m}^{2}\right) \\
& -(n-\sigma) \int \frac{K^{1 / n} f_{\sigma}^{p-1}}{H^{1-\sigma}}\langle\nabla H, \nabla f\rangle_{m}+n(n-\sigma) \int \frac{K^{1 / n} f_{\sigma}^{p-1}}{H^{n+2-\sigma}}|\nabla H|_{m}^{2} \\
& +\frac{1}{n^{n-1}} \int \frac{K^{1 / n} f_{\sigma}^{p-1}}{H^{3-\sigma}}\left|H \nabla_{i} h_{k l}-\nabla_{i} H h_{k l}\right|_{g, h}^{2} \\
& -\frac{2}{n^{n-1}} \int H^{n-2+\sigma} K^{1 / n-1} f_{\sigma}^{p-1}\langle\nabla H, \nabla f\rangle+\frac{1}{n^{n-2}} \int \frac{K^{1 / n} f_{\sigma}^{p-1}}{H^{3-\sigma}}|\nabla H|^{2} \\
& +\frac{n-1}{n^{n-1}} \int H^{n-2+\sigma} K^{1 / n-1} f_{\sigma}^{p-1}\langle\nabla H, \nabla f\rangle+\frac{n-1}{n^{n-1}} \int \frac{K^{1 / n} f_{\sigma}^{p-1}}{H^{2-\sigma}}|\nabla H|_{h}^{2} \\
& -\frac{n-1}{n^{n-2}} \int \frac{K^{1 / n} f_{\sigma}^{p-1}}{H^{3-\sigma}}|\nabla H|^{2}+\frac{1}{n^{n-2}} \int \frac{K^{1 / n} f_{\sigma}^{p-1}}{H^{1-\sigma}}\left(|A|^{2}-\frac{1}{n} H^{2}\right) \\
& -\int \frac{K^{1 / n-1} f_{\sigma}^{p-1}}{H^{n-\sigma}}\left(H^{2 n}|\nabla f|_{h}^{2}-2 n H^{n-1} K\langle\nabla H, \nabla f\rangle_{h}+\frac{n^{2} K^{2}}{H^{2}}|\nabla H|_{h}^{2}\right) \\
& +p(n-\sigma) \int \frac{K^{1 / n} f_{\sigma}^{p-1}}{H}\left\langle\nabla H, \nabla f_{\sigma}\right\rangle_{h} \\
& -\frac{(n-\sigma)}{n} \int H^{n-1} K^{1 / n-1} f_{\sigma}^{p}\left\langle\nabla H, \nabla f_{\sigma}\right\rangle_{h} \\
& +(n-\sigma) \int H^{n-1} K^{1 / n-1} f_{\sigma}^{p}\langle\nabla H, \nabla f\rangle_{h} \\
& -2(n-\sigma) \int \frac{K^{1 / n} f_{\sigma}^{p-1}}{H}\left\langle\nabla H, \nabla f_{\sigma}\right\rangle_{h} \\
& +(n-\sigma)(1-2 n+\sigma) \int \frac{K^{1 / n} f_{\sigma}^{p}}{H^{2}}|\nabla H|_{h}^{2},
\end{aligned}
$$

where $m=\left((H / n)^{n-1} g^{-1}-K h^{-1}\right)^{-1}$. 
Since (31) is rather complicated, we first make some remarks to clarify the situation. There are basically three types of terms in (31): $\left|\nabla f_{\sigma}\right|^{2},\left\langle\nabla H, \nabla f_{\sigma}\right\rangle$ and $|\nabla H|^{2}$ terms. The $p(n-\sigma) \int\left(K^{1 / n} f_{\sigma}^{p-1} / H\right)\left|\left\langle\nabla H, \nabla f_{\sigma}\right\rangle_{h}\right|$ term with the term

$$
n(p-1) \int K^{1 / n} f_{\sigma}^{p-2} H^{-n-1+\sigma}\left|\left\langle\nabla H, \nabla f_{\sigma}\right\rangle_{m}\right|
$$

will dominate the rest of the $\left\langle\nabla H, \nabla f_{\sigma}\right\rangle$ terms by choosing $p$ large enough depending only on $M_{0}$. The $|\nabla H|^{2}$ terms, modulo ones with $\sigma$ 's in the coefficient, are

$$
\begin{aligned}
& -n \int \frac{K^{1 / n} f_{\sigma}^{p-1}}{H^{n+2-\sigma}}|\nabla H|_{m}^{2}+n^{2} \int \frac{K^{1 / n} f_{\sigma}^{p-1}}{H^{n+2-\sigma}}|\nabla H|_{m}^{2} \\
& +\frac{1}{n^{n-2}} \int \frac{K^{1 / n} f_{\sigma}^{p-1}}{H^{3-\sigma}}|\nabla H|^{2}+\frac{n-1}{n^{n-1}} \int \frac{K^{1 / n} f_{\sigma}^{p-1}}{H^{2-\sigma}}|\nabla H|_{h}^{2} \\
& -\frac{n-1}{n^{n-2}} \int \frac{K^{1 / n} f_{\sigma}^{p-1}}{H^{3-\sigma}}|\nabla H|^{2}-\int \frac{K^{1 / n+1}}{H^{n+2-\sigma}} f_{\sigma}^{p-1}|\nabla H|_{h}^{2} \\
& +n(1-2 n) \int \frac{K^{1 / n} f_{\sigma}^{p-1}}{H^{2+n-\sigma}}\left(\frac{1}{n^{n}} H^{n}-K\right)|\nabla H|_{h}^{2} \\
& =\left(-n(n-1)-n^{2}-n(1-2 n)\right) \int \frac{K^{1 / n+1} f_{\sigma}^{p-1}}{H^{n+2-\sigma}}|\nabla H|_{h}^{2} \\
& +\left(\frac{n-1}{n^{n-1}}+\frac{n(1-2 n)}{n^{n}}\right) \int \frac{K^{1 / n} f_{\sigma}^{p-1}}{H^{2-\sigma}}|\nabla H|_{h}^{2} \\
& +\left(\frac{n-1}{n^{n-2}}+\frac{1}{n^{n-2}}-\frac{n-1}{n^{n-2}}\right) \int \frac{K^{1 / n} f_{\sigma}^{p-1}}{H^{3-\sigma}}|\nabla H|^{2} \\
& =\frac{1}{n^{n-2}} \int \frac{K^{1 / n} f_{\sigma}^{p-1}}{H^{2-\sigma}}|\nabla H|_{\left(g^{-1} / H-h^{-1}\right)^{-1}}^{2}
\end{aligned}
$$

The $\left|\nabla f_{\sigma}\right|^{2}$ terms are

$$
\begin{gathered}
(p-1) \int K^{1 / n-1} f_{\sigma}^{p-2}\left|\nabla f_{\sigma}\right|_{m}^{2}-\frac{1}{n} \int H^{n-\sigma} K^{1 / n-2} f_{\sigma}^{p-1}\left|\nabla f_{\sigma}\right|_{m}^{2} \\
-\int H^{n-\sigma} K^{1 / n-1} f_{\sigma}^{p-1}\left|\nabla f_{\sigma}\right|_{h}^{2}
\end{gathered} .
$$


By the preceding remarks and (31), we have

$$
\begin{aligned}
\int K^{1 / n} f_{\sigma}^{p-1} \square & f_{\sigma} \geqslant(p-1) \int K^{1 / n-1} f_{\sigma}^{p-2}\left|\nabla f_{\sigma}\right|_{m}^{2} \\
& -\frac{1}{n} \int H^{n-\sigma} K^{1 / n-2} f_{\sigma}^{p-1}\left|\nabla f_{\sigma}\right|_{m}^{2}-\int H^{n-\sigma} K^{1 / n-1} f_{\sigma}^{p-1}\left|\nabla f_{\sigma}\right|_{h}^{2} \\
& -C_{9} p \int \frac{f_{\sigma}^{p-1}}{H}|\nabla H|\left|\nabla f_{\sigma}\right|-C_{10} \int \frac{f_{\sigma}^{p-1}}{H^{2-\sigma}}|\nabla H|^{2} \\
& -C_{11} p \int H^{-n+\sigma} f_{\sigma}^{p-2}|\nabla H|\left|\nabla f_{\sigma}\right|\left|\left(\frac{H}{n}\right)^{n-1} g_{i j}^{-1}-K h_{i j}^{-1}\right| \\
& +C_{12} \int H^{\sigma} f_{\sigma}^{p-1}\left(|A|^{2}-\frac{1}{n} H^{2}\right),
\end{aligned}
$$

where $C_{9}, C_{10}, C_{11}$ and $C_{12}$ depend only on $M_{0}$.

Combining (28) and (32),

$$
\begin{aligned}
0 \geqslant & (p-1) \int K^{1 / n} f_{\sigma}^{p-2}\left|\nabla f_{\sigma}\right|_{h}^{2}+\left(1-\frac{1}{n}\right) \int H^{n-\sigma} K^{1 / n-1} f_{\sigma}^{p-1}\left|\nabla f_{\sigma}\right|_{h}^{2} \\
& -(n-1) \int \frac{K^{1 / n} f_{\sigma}^{p-1}}{H}\left\langle\nabla H, \nabla f_{\sigma}\right\rangle_{h} \\
& -\sigma\left(1-\frac{1}{n}\right) \int H^{n-1-\sigma} K^{1 / n-1} f_{\sigma}^{p}\left\langle\nabla H, \nabla f_{\sigma}\right\rangle_{h} \\
& +(p-1) \int K^{1 / n-1} f_{\sigma}^{p-2}\left|\nabla f_{\sigma}\right|_{m}^{2} \\
& -\frac{1}{n} \int H^{n-\sigma} K^{1 / n-2} f_{\sigma}^{p-1}\left|\nabla f_{\sigma}\right|_{m}^{2}-\int H^{n-\sigma} K^{1 / n-1} f_{\sigma}^{p-1}\left|\nabla f_{\sigma}\right|_{h}^{2} \\
& -C_{9} p \int \frac{f_{\sigma}^{p-1}}{H}|\nabla H|\left|\nabla f_{\sigma}\right| \\
& -C_{11} p \int H^{-n+\sigma} f_{\sigma}^{p-2}|\nabla H|\left|\nabla f_{\sigma}\right|\left|\left(\frac{H}{n}\right)^{n-1} g^{i j}-K h_{i j}^{-1}\right| \\
& -C_{10} \int \frac{f_{\sigma}^{p-1}}{H^{2-\sigma}}|\nabla H|^{2}+C_{12} \int H^{\sigma} f_{\sigma}^{p-1}\left(|A|^{2}-\frac{1}{n} H^{2}\right) .
\end{aligned}
$$

In order to simplify (33) we first prove a small lemma.

5.5 Lemma. There exist positive constants $C_{13}$ and $C_{14}$ depending only on $n$ such that
(i) $\frac{1}{n^{n}} H^{n}-K \leqslant C_{13} H^{n-2}\left(|A|^{2}-\frac{1}{n} H^{2}\right)$,
(ii) $\left|\left(\frac{H}{n}\right)^{n-1} g^{i j}-K h_{i j}^{-1}\right| \leqslant C_{14} H^{n-1} f^{1 / 2}$. 
Proof. (i) Since both sides are homogeneous of degree $n$, we may assume without loss of generality that $H=\sum_{i=1}^{n} \kappa_{i}=n$. We can then write $\kappa_{i}, i=$ $1, \cdots, n$, uniquely as $\kappa_{i}=1+\varepsilon p_{i}$, where $\varepsilon \geqslant 0, \sum_{i=1}^{n} p_{i}=0$ and $\sum_{i=1}^{n} p_{i}^{2}=1$. Since $\sum_{i \neq j} p_{i} p_{j}=\left(\sum_{i} p_{i}\right)^{2}-\sum_{i} p_{i}^{2}=-1$;

$$
\begin{aligned}
\frac{1}{n^{n}} H^{n}-K & =1-\prod_{i=1}^{n}\left(1+\varepsilon p_{i}\right)=-\varepsilon \sum_{i} p_{i}-\varepsilon^{2}\left(\sum_{i \neq j} p_{i} p_{j}\right)+O\left(\varepsilon^{2}\right) \\
& =\varepsilon^{2}+O\left(\varepsilon^{3}\right) .
\end{aligned}
$$

Also, $H^{n-2}\left(|A|^{2}-H^{2} / n\right)=n^{n-2}\left(2 \varepsilon \sum_{i} p_{i}+\varepsilon^{2} \sum_{i} p_{i}^{2}\right)=n^{n-2} \varepsilon^{2}$. Part (i) now follows.

(ii) If we make the same assumptions as in the proof of part (i), we have

$$
\begin{aligned}
\left|\left(\frac{H}{n}\right)^{n-1} g^{-1}-K h^{-1}\right|^{2} & =\sum_{j=1}^{n}\left(1-\prod_{i \neq j}\left(1+p_{i} \varepsilon\right)\right)^{2} \\
& =\sum_{j=1}^{n} \cdot\left(\left(\sum_{i \neq j} p_{i}\right) \varepsilon+O\left(\varepsilon^{2}\right)\right)=\varepsilon^{2}+O\left(\varepsilon^{3}\right) .
\end{aligned}
$$

This proves part (ii).

Applying Lemma 5.5 to (33) gives, for large enough $p$,

$$
\begin{aligned}
0 \geqslant & C_{15} p \int f_{\sigma}^{p-2}\left|\nabla f_{\sigma}\right|^{2}-C_{16} p \int H^{\sigma / 2-1} f_{\sigma}^{p-3 / 2}|\nabla H|\left|\nabla f_{\sigma}\right| \\
& -C_{10} \int H^{\sigma-2} f_{\sigma}^{p-1}|\nabla H|^{2}+C_{17} \int H^{2} f_{\sigma}^{p},
\end{aligned}
$$

where $C_{10}, C_{15}, C_{16}$ and $C_{17}$ are positive constants depending only on $M_{0}$. Lemma 5.4 now follows from applying the inequality $a b \leqslant \frac{1}{2} \eta a^{2}+\frac{1}{2} b^{2} / \eta$, $\eta>0$.

By Lemmas 5.3 and 5.4, we have by choosing $\eta=p^{-1 / 2}$

$$
\begin{gathered}
\frac{\partial}{\partial t} \int f_{\sigma}^{p} \leqslant-C_{6} p^{2} \int f_{\sigma}^{p-2}\left|\nabla f_{\sigma}\right|^{2}-C_{7} p \int H^{\sigma-2} f_{\sigma}^{p-1}|\nabla H|^{2}+C_{7^{\prime}} \sigma p \int H^{2} f_{\sigma}^{p}, \\
\int H^{2} f_{\sigma}^{p} \leqslant\left(C_{19} p^{1 / 2}+C_{20}\right) \int H^{\sigma-2} f_{\sigma}^{p-1}|\nabla H|^{2}+C_{21} p^{3 / 2} \int f_{\sigma}^{p-2}\left|\nabla f_{\sigma}\right|^{2},
\end{gathered}
$$

which imply

$$
\begin{aligned}
\frac{\partial}{\partial t} \int f_{\sigma}^{p} \leqslant & \left(-C_{6} p^{2}+C_{22} \sigma p^{5 / 2}\right) \int f_{\sigma}^{p-2}\left|\nabla f_{\sigma}\right|^{2} \\
& +\left(-C_{7} p+C_{23} \sigma p^{3 / 2}+C_{24} \sigma p\right) \int H^{\sigma-2} f_{\sigma}^{p-1}|\nabla H|^{2},
\end{aligned}
$$

where the constants depend only on $M_{0}$. 
Therefore, $(\partial / \partial t) \int f_{\sigma}^{p} \leqslant 0$ for $p \geqslant C_{25}$ and $\sigma \leqslant C_{26} p^{-1 / 2}$, where $C_{25}<\infty$ and $C_{26}$ depend only on $M_{0}$. Now that we obtained suitable $L^{p}$ bounds on $f_{\sigma}$, we may continue exactly as in [4] to obtain a supremum bound on $f_{\sigma}$ for $\sigma$ sufficiently small by an iteration argument. This completes the proof of Theorem 5.1.

\section{Tso's Theorem}

In [9], K. Tso studied (III) of $\S 1$ for $\beta=1$. His proof of Theorem 1.2 extends to the case of any $\beta>0$ with only trivial modifications. For completeness, we give a brief exposition of his argument in this section. Tso considered (II) from the point of view of the unnormalized equation and proved $C^{\infty}$. convergence of the $M_{t}$ 's for the unnormalized equation. In the previous sections, we followed Huisken in looking at the metric and curvatures of $M$ to get the zeroth order estimate, but we did not succeed in using his method to get the gradient or higher derivative estimates. It is by combining both methods that we are able to prove Theorem 1.3. We now present Tso's argument (see [9] for details).

We parametrize $M$ by the inverse of the Gauss map which is well defined since $M$ is strictly convex. Then $F: S^{n} \rightarrow M$ takes a unit vector $\nu \in S^{n}$ to the point $F(\nu)$ on $M$ having $\nu$ as its outward normal. The support function $h$ : $S^{n} \rightarrow \mathbf{R}$ is defined by $h(\nu)=\langle F(\nu), \nu\rangle$. (III) is clearly equivalent to the equation

$$
\frac{\partial}{\partial t} h(\nu)=-K(\nu)^{\beta}, \quad \nu \in S^{n} .
$$

It is standard to extend $h$ to $\mathbf{R}^{n+1}$ as a function homogeneous of degree 1 and then consider its restriction to a hyperplane. We therefore define $u: \mathbf{R}^{n} \rightarrow \mathbf{R}$ by $u(x)=h(x,-1), x=\left(x^{1}, \cdots, x^{n}\right) \in \mathbf{R}^{n}$. The Gaussian curvature of $M$ as a function of a normal vector in the southern hemisphere of $S^{n}$ is given by

$$
K(\nu)=\left(1+|x|^{2}\right)^{-(n / 2+1)}\left(\operatorname{det} u_{i j}(x)\right)^{-1}, \quad \nu=(x,-1) / \sqrt{1+|x|^{2}},
$$

where $\left(u_{i j}(x)\right)$ denotes the Hessian of $u$ at $x \in \mathbf{R}^{n}$. In terms of the function $u$, (III) becomes

$$
\begin{gathered}
-u_{t}(x)\left(\operatorname{det} u_{i j}(x)\right)^{\beta}=\left(1+|x|^{2}\right)^{-\beta(n+2) / 2+1 / 2}, \\
u(x, 0)=h_{0}(x,-1), \quad x \in \mathbf{R}^{n},
\end{gathered}
$$

where $h_{0}$ is the support function of $M_{0}$.

As is usual for parabolic equations, the Hölder norms $C^{k, \alpha}\left(S^{n} \times[0, t]\right)$ are defined with time derivatives counting as two space derivatives. We then have 
6.1 Lemma. If $h \in C^{2}\left(S^{n} \times[0, t]\right)$ is a smooth solution of (34) and $h \geqslant r>$ 0 , then there exists a constant $C<\infty$ depending only on $r$ and $M_{0}$ such that

$$
\|h\|_{C^{2}\left(S^{2} \times[0, t]\right)} \leqslant C \text {. }
$$

Proof. We first bound $h$ and $\partial h / \partial t$. By Corollary 4.1, $-\partial h / \partial t \geqslant \inf _{t=0} K^{\beta}$ $>0$ and hence $r \leqslant h \leqslant \sup _{t=0} h$. To bound $-\partial h / \partial t$ from above, Tso considered the function $\varphi(\nu)=-(\partial h / \partial t)(\nu) /\left(h(\nu)-r^{\prime}\right), \nu \in S^{n}$, and where $r^{\prime}<$ $r$. If $\varphi$ attains its maximum at $\left(\nu_{1}, t_{1}\right)$ with $t_{1}>0$, then by applying standard maximum principle arguments, we derive $H \leqslant(n+1 / p) / r^{\prime}$ at $\left(\nu_{1}, t_{1}\right)$. The bound $-\partial h / \partial t \leqslant C$ then follows. The bound on $|\nabla h|$ follows from the bound on $h$ and the convexity of $u$. To bound $\left|\nabla^{2} h\right|$ we apply the maximum principle to the function

$$
\psi=\frac{\partial^{2} u}{\partial x_{1}^{2}} \frac{\left(1+|x|^{2}\right)^{3 / 2}}{\left(1+|x|^{2}-x_{1}^{2}\right)},
$$

where we have assumed without loss of generality $h_{\alpha \beta}$ is diagonal and achieves its maximum $h_{11}$ at $\left(0,-1, t_{1}\right), 0=(0, \cdots, 0) \in \mathbf{R}^{n}$ and $t_{1}>0$. Then, as in [9], we have $R \leqslant\left(-\frac{1}{2}(n+2)+1 / 2 \beta\right) H^{-1} \leqslant C$ at $\left(0,-1, t_{1}\right)$. The upper bound for $h_{\alpha \beta}$ follows. Since $K \leqslant C$, we also have a lower bound for $h_{\alpha \beta}$. By the preceding remarks, we have $\|h\|_{C^{2}\left(S^{n} \times[0, t]\right)} \leqslant C\left(r, M_{0}\right)$. This completes the proof of Lemma 6.1.

The $C^{2, \alpha}$ estimate of $h$ now follows from a theorem of Krylov-Safonov [10] and an adaptation of an argument due to Trudinger [8] (again see [9] for details). The $C^{\infty}$ estimate for $h$ now follows from standard arguments. By applying the previous estimates to the normalized equation, we may show $C^{\infty}$-convergence to a hypersurface $\tilde{M}_{T}$ for the normalized equation.

\section{Conclusion}

We now show how $C^{\infty}$-convergence to a convex hypersurface $\tilde{M}_{T}$ and the estimate $f=\left((H / n)^{n}-K\right) / H^{n} \leqslant C H^{-\sigma}$ for some $\sigma>0$ implies Theorem 1.3. Clearly there is some point $p$ on $\tilde{M}_{T}$ with $K(p)>0$; this is true for any hypersurface by taking the point farthest from the origin. By continuity there is a neighborhood $U$ of $p$ with $K>0$ in $U$. Then, for the unnormalized equation, $H(x)$ approaches infinity for every $x \in U$. By scale the invariance of $f$, $f(x)=0$ for $x \in U \subset \tilde{M}_{T}$. Therefore every point $x \in U$ is an umbilic point and by a standard result, $K$ is constant in $U$. Clearly, $K$ must then be constant on $\tilde{M}_{T}$ and hence $\tilde{M}_{T}$ is a round sphere. This completes the proof of Theorem 1.3. 


\section{References}

[1] W. J. Firey, Shapes of worn stones, Mathematika 21 (1974) 1-11.

[2] M. Gage \& R. S. Hamilton, The heat equation shrinking convex plane curves, J. Differential Geometry, to appear.

[3] R. S. Hamilton, Three-manifolds with positive Ricci curvature, J. Differential Geometry 17 (1982) 255-306.

[4] G. Huisken, Flow by mean curvature of convex surfaces into spheres, J. Differential Geometry 20 (1984) 237-266.

[5] _ Ricci deformation of the metric on a Riemannian manifold, J. Differential Geometry, to appear.

[6] J. H. Michael \& L. M. Simon, Sobolev and mean value inequalities on generalized submanifolds of $\mathbf{R}^{\prime \prime}$, Comm. Pure Appl. Math. 26 (1973) 316-379.

[7] G. Stampacchia, Equations elliptiques au second order à coéfficients discontinues, Sém. Math. Sup. 16, Les Presses de l'Université de Montreal, 1966.

[8] N. S. Trudinger, Elliptic equations in nondivergence form, Proc. Miniconference on Partial Differential Equations, Canberra, 1981, 1-16.

[9] K. Tso, Deforming a hypersurface by its Gauss-Kronecker curvature, Comm. Pure Appl. Math., to appear.

[10] N. V. Kyrlov \& M. V. Safonov, A certain property of solutions of parabolic equation with measurable coefficients, Math. USSR Izv. 16 (1981) 151-164.

University of California at San Diego 